\title{
Antidiabetic Biguanides Radiosensitize Hypoxic Colorectal Cancer Cells Through a Decrease in Oxygen Consumption
}

\section{OPEN ACCESS}

Edited by:

Olivier Cuvillier,

Centre National de la Recherche Scientifique (CNRS), France

Reviewed by:

Chris R. Triggle,

Weill Cornell Medicine-Qatar, Qatar

Lucas Sullivan,

Fred Hutchinson Cancer Research

Center, United States

${ }^{*}$ Correspondence: Mark De Ridder

mark.deridder@uzbrussel.be

Specialty section:

This article was submitted to Pharmacology of Anti-Cancer Drugs,

a section of the journal

Frontiers in Pharmacology

Received: 15 June 2018 Accepted: 05 September 2018 Published: 03 October 2018

Citation:

de Mey S, Jiang $H$, Corbet $C$ Wang H, Dufait I, Law K, Bastien E, Verovski V, Gevaert T, Feron $O$ and

De Ridder M (2018) Antidiabetic Biguanides Radiosensitize Hypoxic

Colorectal Cancer Cells Through a Decrease in Oxygen Consumption.

Front. Pharmacol. 9:1073. doi: 10.3389/fphar.2018.01073

\author{
Sven de Mey ${ }^{1}$, Heng Jiang ${ }^{1}$, Cyril Corbet ${ }^{2}$, Hui Wang ${ }^{1}$, Inès Dufait ${ }^{1,3}$, Kalun Law ${ }^{1}$, \\ Estelle Bastien" ${ }^{2}$, Valeri Verovski ${ }^{1}$, Thierry Gevaert ${ }^{1}$, Olivier Feron ${ }^{2}$ and Mark De Ridder ${ }^{1 *}$ \\ ${ }^{1}$ Department of Radiotherapy, Universitair Ziekenhuis Brussel, Vrije Universiteit Brussel, Brussels, Belgium, ${ }^{2}$ Pole \\ of Pharmacology and Therapeutics, Institut de Recherche Expérimentale et Clinique (IREC), Université catholique \\ de Louvain, Brussels, Belgium, ${ }^{3}$ Laboratory of Molecular and Cellular Therapy, Vrije Universiteit Brussel, Brussels, Belgium
}

Background and Purpose: The anti-diabetic biguanide drugs metformin and phenformin exhibit antitumor activity in various models. However, their radiomodulatory effect under hypoxic conditions, particularly for phenformin, is largely unknown. This study therefore examines whether metformin and phenformin as mitochondrial complex I blockades could overcome hypoxic radioresistance through inhibition of oxygen consumption.

Materials and Methods: A panel of colorectal cancer cells (HCT116, DLD-1, HT29, SW480, and CT26) was exposed to metformin or phenformin for $16 \mathrm{~h}$ at indicated concentrations. Afterward, cell viability was measured by MTT and colony formation assays. Apoptosis and reactive oxygen species (ROS) were detected by flow cytometry. Phosphorylation of AMP-activated protein kinase (AMPK) was examined by western blot. Mitochondria complexes activity and oxygen consumption rate (OCR) were measured by seahorse analyzer. The radiosensitivity of tumor cells was assessed by colony formation assay under aerobic and hypoxic conditions. The in vitro findings were further validated in colorectal CT26 tumor model.

Results: Metformin and phenformin inhibited mitochondrial complex I activity and subsequently reduced OCR in a dose-dependent manner starting at $3 \mathrm{mM}$ and $30 \mu \mathrm{M}$, respectively. As a result, the hypoxic radioresistance of tumor cells was counteracted by metformin and phenformin with an enhancement ratio about 2 at $9 \mathrm{mM}$ and $100 \mu \mathrm{M}$, respectively. Regarding intrinsic radioresistance, both of them did not exhibit any effect although there was an increase of phosphorylation of AMPK and ROS production. In tumor-bearing mice, metformin or phenformin alone did not show any anti-tumor effect. While in combination with radiation, both of them substantially delayed tumor growth and enhanced radioresponse, respectively, by 1.3 and 1.5-fold.

Conclusion: Our results demonstrate that metformin and phenformin overcome hypoxic radioresistance through inhibition of mitochondrial respiration, and provide a rationale to explore metformin and phenformin as hypoxic radiosensitizers.

Keywords: phenformin, metformin, hypoxic radiosensitivity, mitochondrial complex I, oxygen consumption rate, colorectal cancer 


\section{INTRODUCTION}

Metformin, an oral biguanide, is the most widely used drug to treat type II diabetes. Epidemiological evidence indicates that metformin reduces cancer risk and enhances response to therapeutics, consequently decreasing cancer-related mortality (Decensi et al., 2010; Quinn et al., 2013). Currently, repurposing metformin as an anti-cancer drug is receiving considerable attention with over 100 clinical trials, wherein its anti-cancer effect is explored alone or combined with other therapies (Chae et al., 2016). The enthusiasm toward repurposing metformin is extended to another biguanide anti-diabetic drug, namely phenformin. As a therapeutic for diabetes, its use has been limited to relatively few countries due to an increased incidence of lactic acidosis in elderly patients with renal failure (0.4-0.64 cases per 1,000 patient) (Pernicova and Korbonits, 2014; Liu et al., 2015). While as a potential anti-cancer drug, phenformin showed much higher cytotoxic activity toward tumor- and tumor-supporting cells compared with metformin in various preclinical models (Shackelford et al., 2013; Gravel et al., 2014; Velez et al., 2016).

The mechanisms by which metformin and phenformin inhibit cancer development and tumor growth are not completely elucidated; whereas emerging evidence suggests that inhibition of mitochondrial complex I is one of the major mechanisms. Firstly, inhibition of complex I by metformin and phenformin is associated with an increased cancer cell susceptibility to apoptosis via changing the topology of the inner mitochondrial membrane (Velez et al., 2016). Secondly, inhibition of complex I increases the aberrant flow of electrons to oxygen and creates reactive oxygen species (ROS), subsequently causing damage to proteins, lipids, and nucleic acids (Miskimins et al., 2014). Consistently, metformin and phenformin eliminate tumor cells and tumor-supporting cells such as myeloid-derived suppressor cells (MDSC) through overproduction of ROS (Haugrud et al., 2014; Miskimins et al., 2014; Kim et al., 2017). Thirdly, inhibition of complex I results in impaired mitochondrial metabolism. Thus, the cytotoxic effect of metformin and phenformin is particularly vigorous in cancer cells with a defect in oxidative phosphorylation or glucose uptake (Shackelford et al., 2013; Momcilovic et al., 2015). Intriguingly, perturbation of metabolism by phenformin is reported to reduce stem cell features, leading to decreased cell viability and invasion (Petrachi et al., 2017). Mitochondrial complex I inhibition is an important action by which metformin and phenformin reduce tumor burden, but other mechanisms underlying the anti-cancer effects should not be neglected. Attributed to the antidiabetic properties, metformin reduces circulating glucose and insulin, resulting in a decrease in cell proliferation and invasion; this is particularly effective in tumor with high expression of insulin receptors, such as breast cancer (Goodwin et al., 2012; Mallik and Chowdhury, 2018). In addition, metformin and phenformin trigger the activation of AMP-activated protein kinase (AMPK) that in turn antagonizes the activity of protumor mammalian target of rapamycin (mTOR) signaling, leading to delayed tumor growth and reduced metastasis (Lin et al., 2015; Orecchioni et al., 2015; Navarro et al., 2016).
Complex I is the gatekeeper of the respiratory chain and its inhibition will subsequently result in a decreased oxygen consumption rate (OCR), which is currently considered as a potent strategy to counteract hypoxic radioresistance due to reoxygenation of hypoxic tumor cells (Jiang et al., 2013; Zannella et al., 2013). Hypoxia is a common feature of the tumor microenvironment and considered to be one of the principle causes of clinical failure of radiotherapy (Gray et al., 1953). complex I inhibitors such as arsenic trioxide exhibited clear radiosensitizing effect in solid tumors via increasing tumor oxygenation (Paul et al., 2008; Diepart et al., 2012). Metformin and phenformin have been both characterized as complex I inhibitors and reported to markedly block cellular respiration in diverse tumor cells (Wheaton et al., 2014; Ashton et al., 2016), while their hypoxic radiosensitizing effect, particularly for phenformin, is still largely unexplored. Regarding their impact on aerobic (intrinsic) radiosensitivity, both of them have been reported to radiosensitize aerobic tumor cells, such as lung and breast cancer cells (Storozhuk et al., 2013; Zhang et al., 2014; Wang et al., 2015a), however, this effect is not yet well defined in colorectal cancer (CRC) - the research focus in our department. In addition, the difference in their radiosensitizing potency is almost unknown, even though their cytotoxic effects have been intensively studied in parallel due to the similarity of chemical structure and the common molecular target. In this study, we therefore examined the radiomodulatory effect of metformin and phenform in a panel of CRC cells and further validated our findings in tumor-bearing mice.

\section{MATERIALS AND METHODS}

\section{Cell Lines and Chemicals}

Murine CT26 and human HCT116, DLD-1, HT29, and SW480 CRC cell lines were obtained from American Type Culture Collection (ATCC, Manassas, United States). All experiments were performed in RPMI 1640 medium (Thermo Fisher, Belgium) supplemented with $10 \%$ bovine calf serum (Greiner Bio-One, Belgium). Chemicals were obtained from SigmaAldrich (Antwerp, Belgium) unless otherwise stated.

\section{Treatments}

Cells were grown to subconfluence and exposed to metformin or phenformin (Santa Cruz Biotechnology, Dallas, TX, United States) for $16 \mathrm{~h}$ at indicated concentrations. The ROS scavenger $\mathrm{N}$-acetyl cysteine (NAC) was added at $10 \mathrm{mM}$ to cultures both $1 \mathrm{~h}$ prior and during treatment with metformin or phenformin. Afterward, cultures were used for further analysis as described below.

\section{MTT Assay}

Cytotoxicity of metformin and phenformin was assessed by MTT assay as described elsewhere (Wang et al., 2017). Briefly, after treatment, medium was aspirated and $50 \mu \mathrm{l}$ MTT solution $(5 \mathrm{mg} / \mathrm{ml})$ was added for $1.5 \mathrm{~h}$. Afterward, $200 \mu \mathrm{l}$ of MTT solvent (19:1 DMSO: HCL) was added and admixed to dissolve the formazan crystals generated inside of cells. 
Absorbance was measured at a wavelength of $540 \mathrm{~nm}$ by using a spectrophotometer (Bio-Rad, CA, United States). Cell viability was determined by dividing the absorbance values of treated cells to that of untreated (control) cells.

\section{Apoptotic Assay}

Apoptosis was analyzed by flow cytometry using the double staining with lipophilic Annexin $\mathrm{V}$ and 7-amino actinomycin D (7-AAD) (Abcam, Cambridge, United Kingdom), as described elsewhere (Wang et al., 2017). Briefly, after treatment, cells were harvested and washed with FACS buffer. Thereafter, cells were resuspended in $100 \mu \mathrm{l}$ binding buffer (eBioscience) with $2.5 \mu \mathrm{l}$ Annexin $\mathrm{V}$ (eBioscience) and incubated for $20 \mathrm{~min}$ at room temperature. 7-AAD $(5 \mu \mathrm{l})$ was added $5 \mathrm{~min}$ before analysis. Early apoptotic cells (Annexin V-positive, 7-AAD-negative), necrotic/late apoptotic cells (double-positive), and living cells (double-negative) were determined by flow cytometry (BD LSR Fortsessa, BD Bioscience, Franklin Lakes, NJ, United States).

\section{Radiation and Clonogenic Assay}

After treatment, cells were either subjected to metabolic hypoxia in a micropellet model (Janssens et al., 1998, 1999) or irradiated in suspension. Briefly, to generate micropellets, $0.5 \times 10^{6}$ cells were collected into a $15 \mathrm{ml}$ conical tube and centrifuged at $2000 \mathrm{rpm}$ with a volume of $100 \mu \mathrm{l}$. Then just before radiation, the micropellets were placed in a $37^{\circ} \mathrm{C}$ water bath for $5 \mathrm{~min}$ in order to metabolically consume oxygen and induce hypoxia. For cell suspension, $0.5 \times 10^{6}$ cells were collected into a $15 \mathrm{ml}$ conical tube and suspended into $1 \times 10^{6} / \mathrm{ml}$, and then vortexed and placed in a $37^{\circ} \mathrm{C}$ water bath just before radiation. Afterward, radiation was conducted by using a $6 \mathrm{MV}$ Linac (Elekta, Crowley, United Kingdom) at a rate of $2 \mathrm{~Gy} / \mathrm{min}$; cells were reseeded for colony formation, as described elsewhere (Jiang et al., 2010). After 7-12 days, cultures were fixed with crystal violet and colonies ( $>50$ cells) were counted. Survival curves were fitted to the linear quadratic model using GraphPad Prism 6 software (GraphPad Prism Software Inc., La Jolla, CA, United States). Radiosensitization was expressed as an enhancement ratio determined at a survival fractions (SF) of 0.1 .

\section{Mitochondrial Complexes Activity}

After treatment, mitochondria were isolated from cancer cells and then seeded into a 96 well plate. Afterward, the activity of mitochondrial complexes (I, II, III, and IV) was determined by Seahorse XF96 analyzer (Agilent, Belgium) as depicted in Supplementary Figure $\mathbf{1}$ and described elsewhere (Corbet et al., 2016). Briefly, the mitochondrial complexes activity is reflected by the change of OCR in different phases, following the injection of distinct compounds: $10 \mathrm{mM}$ pyruvate, $5 \mathrm{mM}$ malate, $2 \mathrm{mM}$ $\mathrm{ADP}, 1 \mu \mathrm{M}$ rotenone, $10 \mathrm{mM}$ succinate, $4 \mu \mathrm{M}$ antimycin $\mathrm{A}$, and $10 \mathrm{mM}$ ascorbate plus $0.1 \mathrm{mM}$ TMPD.

\section{Oxygen Consumption Rates}

Oxygen consumption rate was determined by Seahorse XF96 analyzer (Agilent, Belgium). Briefly, $2.0 \times 10^{5}$ cells were seeded in 96-well plates. Twenty-four hours later, OCR of the cells was measured in real-time for $8 \mathrm{~h}$ after injection of various concentrations of metformin or phenformin. OCR was normalized to the basal level.

\section{ROS Production}

The intracellular level of ROS was detected by flow cytometry using an oxidation sensitive fluorescent probe 5-(6)chloromethyl-2', $7^{\prime}$-dichlorodihydro-fluorescein diacetate (CM-H2DCFDA) (Abcam, Cambridge, United Kingdom), as described elsewhere (Wang et al., 2017). Briefly, cells were treated with metformin or phenformin for $16 \mathrm{~h}$, stained with $5 \mu \mathrm{M}(\mathrm{CM}-\mathrm{H} 2 \mathrm{DCFDA})$ at $37^{\circ} \mathrm{C}$ for $30 \mathrm{~min}$ and analyzed by flow cytometry.

\section{Western Blotting}

Western blot was performed as described elsewhere (Jiang et al., 2013). Briefly, cells were lysed by sample buffer (Tris-HCL: $62.5 \mathrm{mM}$ pH 6.8, glycerol: $10 \%$, SDS: $2 \%, \beta$-mercaptoethanol: 5\%, Bromophenol: 0.025\%). Proteins were then separated on a $12.5 \%$ SDS-polyacrylamide gel (Bio-Rad, Hercules, CA, United States) and transferred to nitro-cellulose membrane. Thereafter, membranes were incubated with primary antibodies against AMPK (cell signaling, 5831), phospho-AMPK (cell signaling 2535), and anti-beta actin (cell signaling, 4970). Later, membranes were washed and incubated with appropriate horseradish peroxidase (HRP)-conjugated secondary antibody. Image was taken using the Odyssey Fc imaging system (LI-COR, Belgium).

\section{Mouse Tumor Model}

CT26 tumor cells $\left(0.5 \times 10^{6}\right)$ were inoculated into the left hind limb of syngeneic Balb/c mice (female, 7-9 weeks old; Charles River Laboratories, L'Arbresle Cedex, France). At day 7, mice were randomized and treated with metformin $(300 \mathrm{mg} / \mathrm{kg}$ ) or phenformin $(200 \mathrm{mg} / \mathrm{kg})$ through oral gavage for 10 days. Radiation (9Gy) was delivered by using a 6 MV Linac (Elekta, Crowley, United Kingdom) $1 \mathrm{~h}$ after the first administration of metformin or phenformin. The tumor volume was calculated using the formula Volume $=\left(\text { Length }^{*} \text { Width }^{2}\right)^{*} 0.5$. Experiments were reviewed by the Ethical Committee for use of laboratory animals of the Vrije Universiteit Brussel.

\section{Statistics}

All analyses were performed using prism 6.01 (GraphPad, La Jolla, CA, United States). One-tailed $t$-test, one-way ANOVA followed by a Dunnett's multiple comparison test and twoway ANOVA with Bonferroni's multiple comparison test were performed. Sample sizes and number of repetitions were indicated in the figure legends.

\section{RESULTS}

\section{Cytotoxicity of Metformin and Phenformin Toward a Panel of CRC Cells}

The cytotoxic properties of metformin and phenformin were determined by MTT in a panel of CRC cells. Phenformin 


\section{Cell viability}

A

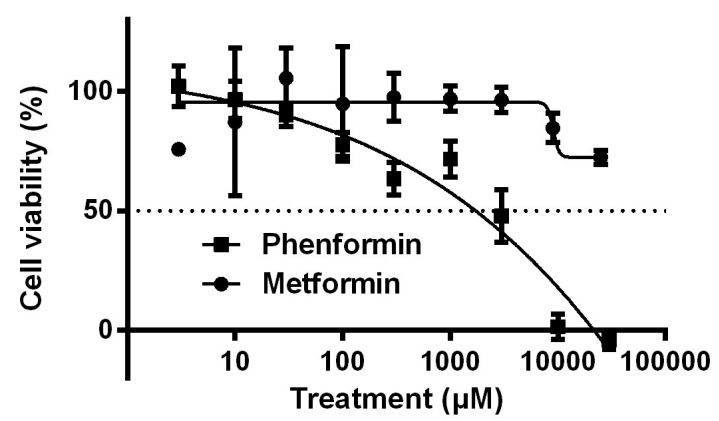

HCT116

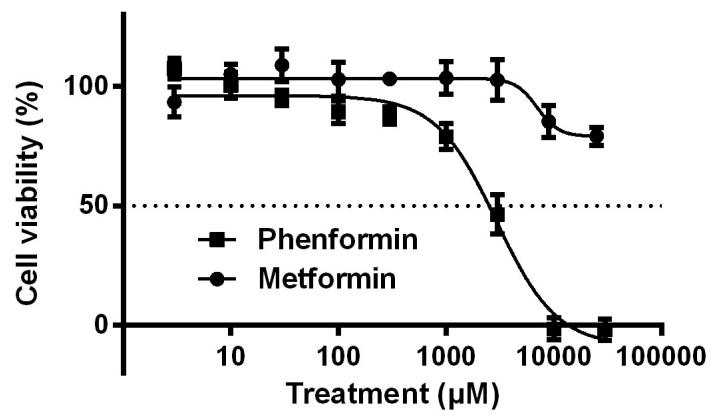

\section{Apoptotic rate}

B

CT26

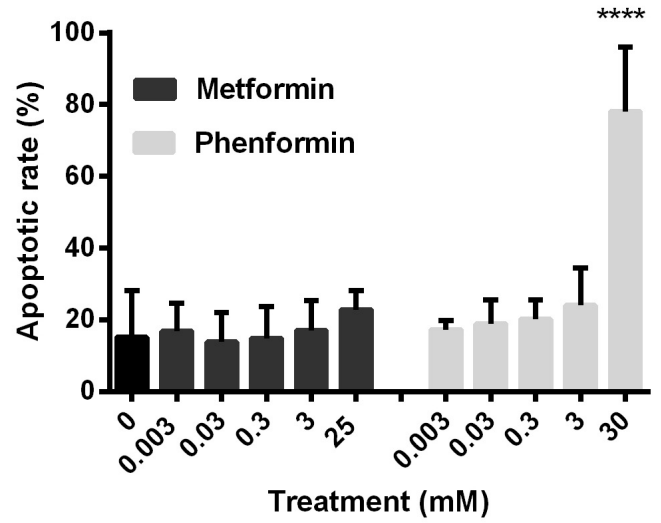

HCT116

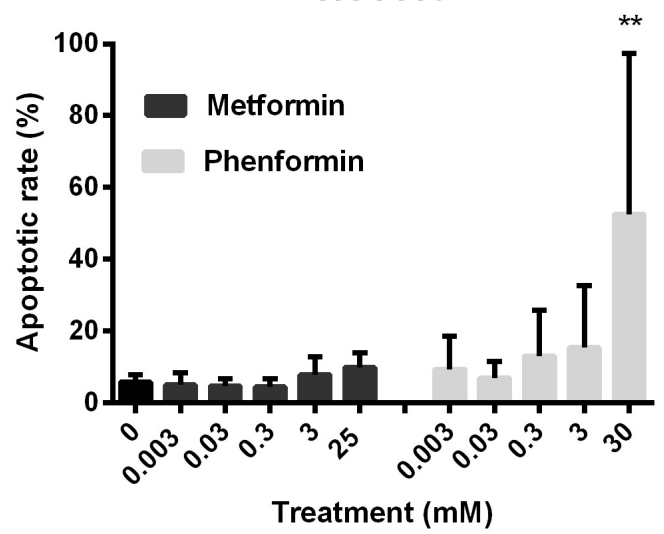

Complex I - dependent OCR

C

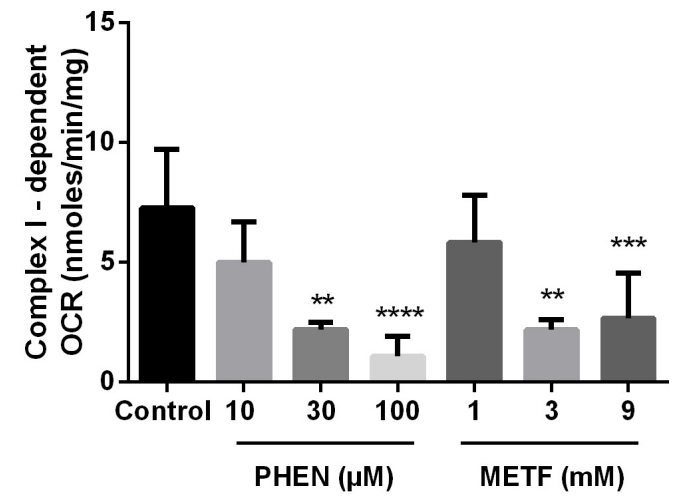

HCT116

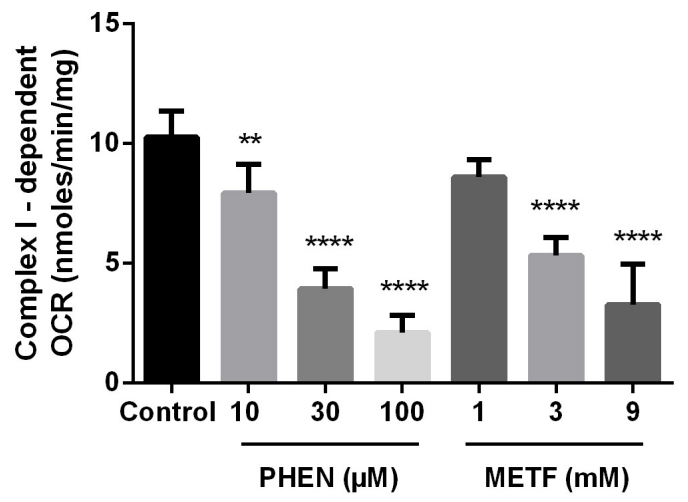

FIGURE 1 | Metformin and phenformin inhibit mitochondrial complex I activity at non-toxic concentrations. CT26 and HCT116 tumor cells were treated with metformin (METF) or phenformin (PHEN) with indicated concentrations for $16 \mathrm{~h}$. Cytotoxicity was determined by using MTT assay (A). Apoptosis was assessed by flow cytometry using Annexin V/7-AAD staining (B). Mitochondria were isolated and activity of mitochondrial complexes I was measured by using a Seahorse analyzer (C). Data are shown from at least three replicates as mean \pm SEM. A one-way ANOVA with Dunnett's multiple comparison test was used to calculate statistics: $* p<0.05,{ }^{* *} p<0.01, * * * p<0.001, * * * p<0.0001$. 


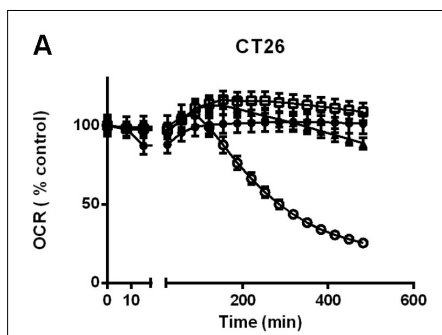

D

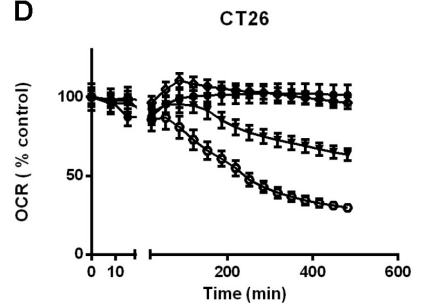

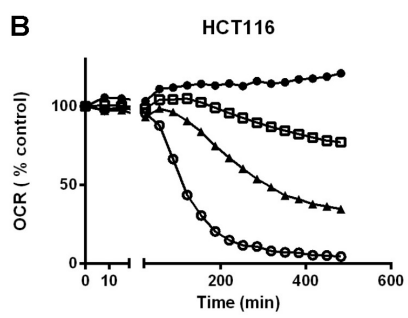

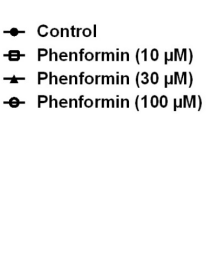

E

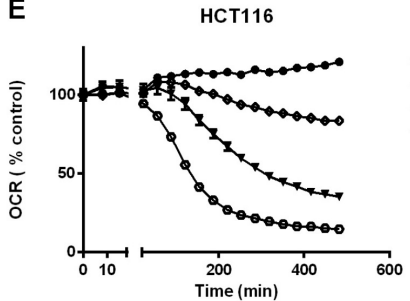

C

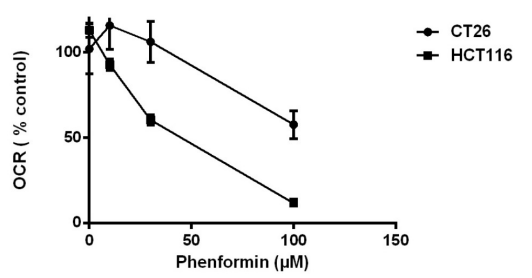

$\mathbf{F}$

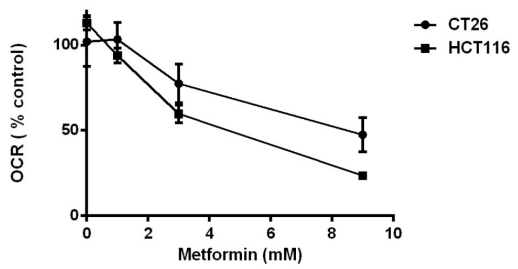

FIGURE 2 | Metformin and phenformin inhibit oxygen consumption. The oxygen consumption rate (OCR) of CT26 (A,D) and HCT116 (B,E) was measured over time after injection of indicated concentrations of metformin or phenformin using the Seahorse analyzer. The OCR was expressed as a percentage relative to control. Relative OCR compared across the two cell lines at $3 \mathrm{~h}$ post-phenformin $\mathbf{( C )}$ or metformin $\mathbf{( F )}$ injection. Data is shown as mean \pm SEM.

reduced cell viability in a dose-dependent manner with IC50 values of $2.9-5.2 \mathrm{mM}$, wherein SW480 is the most sensitive followed by HCT116, CT26, DLD-1, and HT29 (Figure 1A and Supplementary Figure 2A). At high concentrations ( $>3 \mathrm{mM})$, a strong induction of apoptosis, mainly late phase, was detected by flow cytometry (Figure 1B and Supplementary Figure 2B). Oppositely, no considerable effect of metformin on cell viability was observed at doses up to $10 \mathrm{mM}$ (Figures 1A,B and Supplementary Figures 2A,B). At this point, all four human CRC cell lines demonstrated similar cytotoxic profiles after exposure to metformin and phenformin. For practical reasons the subsequent experiments were limited to one murine and one human cell line: namely CT26 and HCT116.

\section{Metformin and Phenformin Inhibited Complex I Activity and Impaired Oxygen Consumption}

At non-toxic doses, we then evaluated the effect of metformin and phenformin on mitochondrial complexes activity by using a Seahorse analyzer. Phenformin inhibited complex I activity with a dose starting at $10 \mu \mathrm{M}$ and reaching a more than $50 \%$ inhibition at $100 \mu \mathrm{M}$ in both CT26 and HCT116 (Figure 1C). Mitochondrial complex II, III and IV were unaffected by phenformin in both cell lines (Supplementary Figures 3A-C). Consequently, phenformin caused a time-dependent decrease in OCR in CT26 at a dose of $100 \mu \mathrm{M}$ (Figure 2A). Compared with CT26, HCT116 is more sensitive to phenformin with a significant inhibition of OCR at a dose of $30 \mu \mathrm{M}$ (Figure 2B). The difference between these two cell lines in response to phenformin was further summarized in Figure 2C. Similarly, metformin specifically inhibited the activity of complex I and thereby time and dose dependently decreased cellular respiration in both CT26 and HCT116 (Figures 1C, 2D-F). However, this effect of metformin required concentrations $>1 \mathrm{mM}$, indicating that phenformin is much more potent than metformin to modulate mitochondrial activity.

\section{Metformin and Phenformin Radiosensitized Hypoxic Tumor Cells}

Decrease of OCR is a potent strategy to reduce tumor hypoxia and overcome hypoxia-induced radioresistance (Secomb et al., 1995; Diepart et al., 2012; Jiang et al., 2013; Lin and Maity, 2015; Zhou et al., 2016). We therefore asked whether metformin and phenformin could radiosensitize hypoxic tumor cells. Radiation experiments were performed in micropellets, a simplified metabolic hypoxia model used to prove the concept. Compared with normoxia, we indeed found a severely impaired radioresponse, with oxygen enhanced ratio of 2.29 and 2.45 for CT26 and HCT116 tumor cells, respectively (Figure 3A), indicating the existence of a deep hypoxia. In line with profound oxygen sparing (Figures 2A,B), phenformin overcame hypoxic radioresistance with enhancement ratios of 1.75 and 2.87 at $100 \mu \mathrm{M}$ for CT26 and HCT116 tumor cells (Figure 3B). With respect to metformin, it improved the hypoxic radiosensitivity of CT26 and HCT116, respectively, by 1.72- and 2.86-fold at a dose of $9 \mathrm{mM}$, a 90 times higher concentration than phenformin (Figure 3C). In addition to tumor hypoxia, intrinsic radioresistance of tumor cells limits the efficacy of radiotherapy as well. Previously, both metformin and phenformin have shown to improve the intrinsic radiosensitivity of tumor cells through activation of the AMPK pathway (Wang et al., 2015a) and overproduction of ROS (Miskimins et al., 2014; Zhang et al., 2014). In our settings, although metformin and phenformin upregulated the phosphorylation of AMPK and induced the production of ROS, no enhanced intrinsic radiosensitivity could be detected under aerobic conditions (Figures 4A-D), suggesting that the intrinsic radiosensitizing effect of metformin and phenformin is cell line dependent. 
A

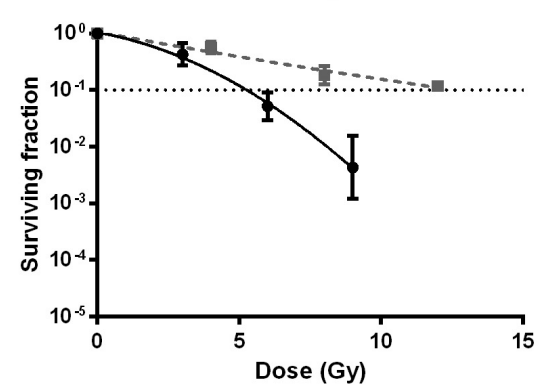

HCT116

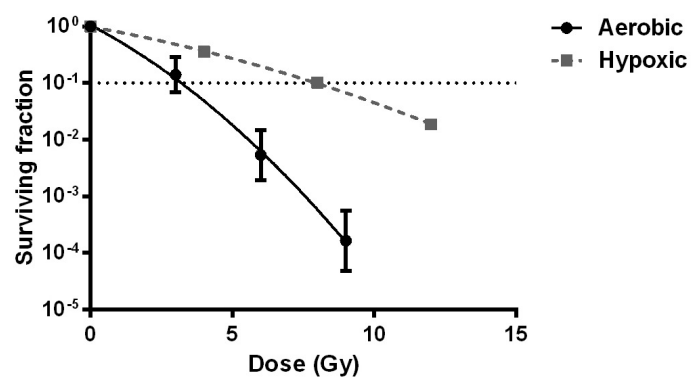

Hypoxic condition

B

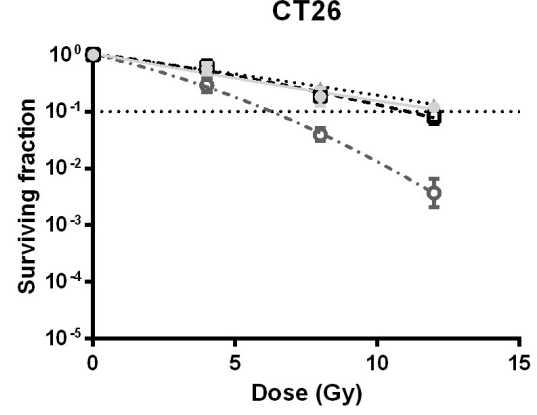

C

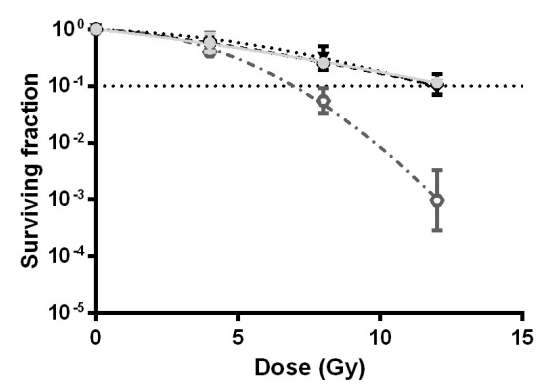

HCT116

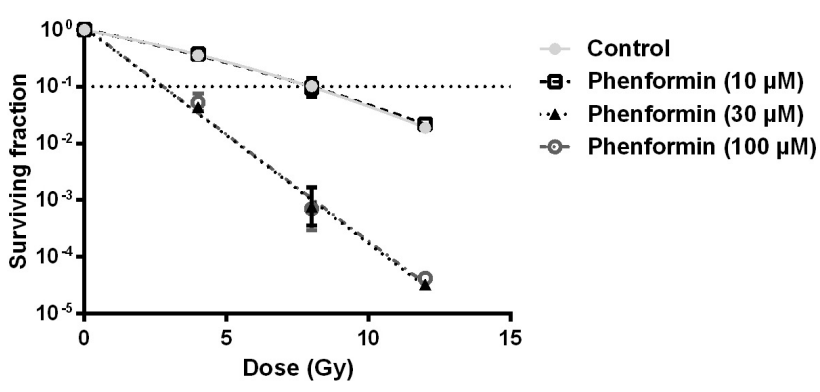

HCT116

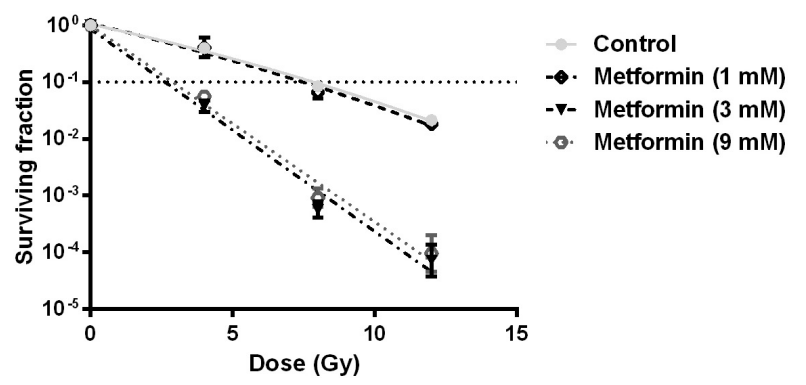

FIGURE 3 | Metformin and phenformin radiosensitize hypoxic tumor cells. CT26 and HCT116 tumor cells were treated with metformin or phenformin for 16 h at indicated concentrations. Afterward, cells were subjected to metabolic hypoxia and irradiated. The radiosensitivity of CT26 and HCT116 tumor cells under either aerobic or hypoxic conditions (A). The radiosensitizing effect of phenformin (B) and metformin (C) under hypoxic conditions was assessed by colony formation assay. Data are shown from at least three experiments as mean $\pm \mathrm{SEM}$.

\section{Metformin and Phenformin Enhanced Radioresponse of CT26 Tumors}

The in vitro findings were further validated in CT26 tumorbearing mice. Radiation alone at 9Gy delayed tumor growth by 17 days, measured at a tumor volume of $1000 \mathrm{~mm}^{3}$ (Figures 5A,D). Metformin (300 $\mathrm{mg} / \mathrm{kg})$ or phenformin (200 mg/kg) alone did not show any anti-tumor effect. While combined with radiation, both of them substantially delayed tumor growth by 26 and 40 days and enhanced radioresponse by 1.3 - and 1.5-fold, respectively. As a result, the medium survival rate of tumor-bearing mice was significantly increased after treated with radiation in combination with metformin or phenformin (Figures 5B,E). Importantly, metformin or phenformin applied orally for 10 days was safe without inducing noticeable toxicity (Figures 5C,F). Altogether, in accordance with the in vitro findings, metformin and phenformin are effective in overcoming hypoxic radioresistance and improving radioresponse of colorectal tumor.

\section{DISCUSSION}

Preclinical evidence suggests that anti-diabetic drug metformin and phenformin can efficiently eliminate cancer cells and reduce metastases as a consequence of inhibition of mitochondrial 


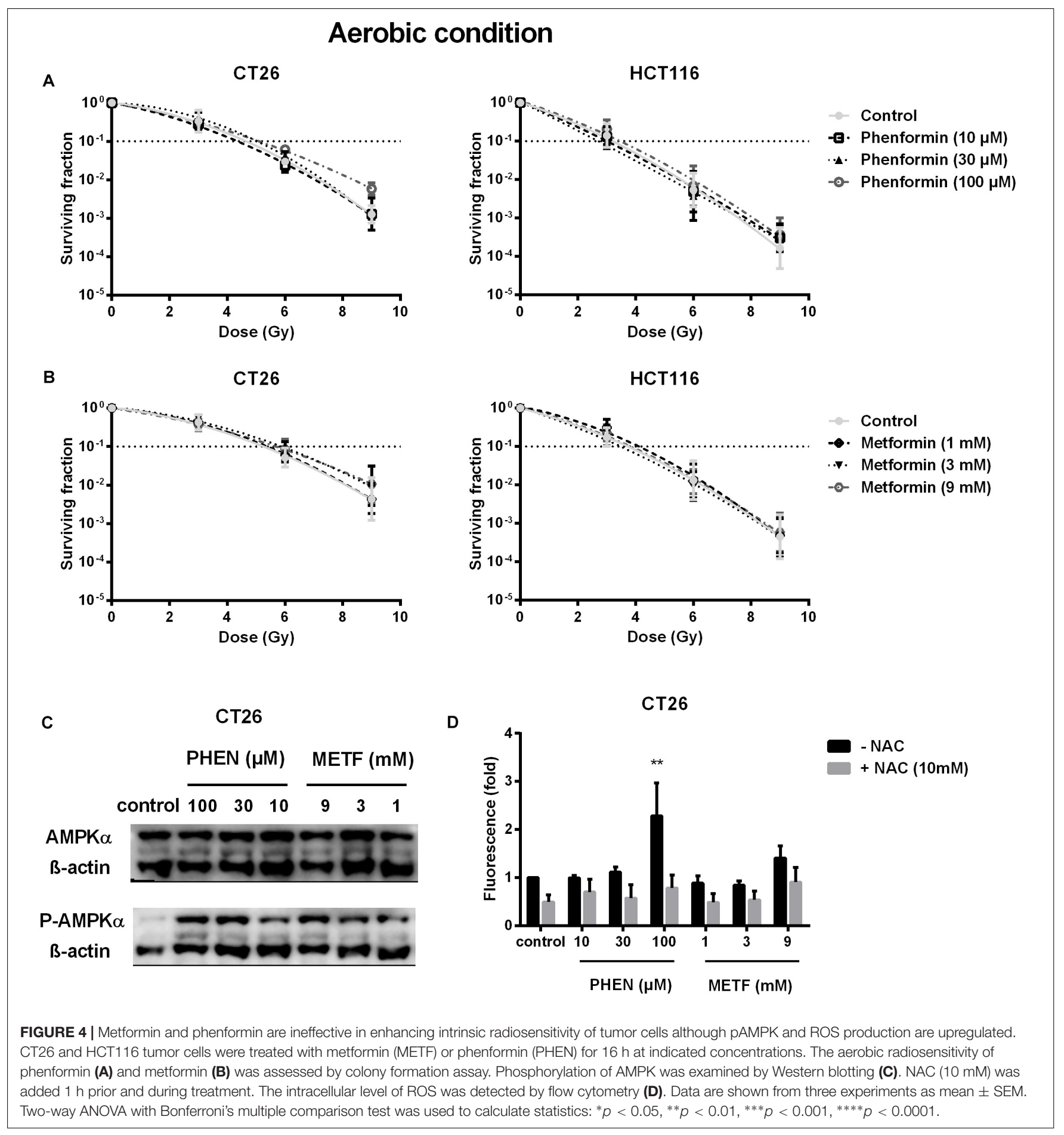

complex I and activation of the AMPK signaling pathway. Next to the direct cytotoxic effect, our study demonstrates that metformin and phenformin are able to overcome hypoxiainduced radioresistance through inhibition of mitochondrial complex I, wherein phenformin exhibited a greater effect than metformin.

Complex I is a rate limiting step for electron transport chain in mitochondria, driving the synthesis of energy-carrying molecule
ATP and in the meantime consumption of oxygen (Matsuzaki and Humphries, 2015). Inhibition of complex I as one of the anticancer mechanisms of metformin and phenformin has drawn quite some attention in the last several years (Andrzejewski et al., 2014; Miskimins et al., 2014; Boukalova et al., 2016; Velez et al., 2016). Whereas about two decades ago, this action of metformin has already been reported in hepatocytes, which contributes to the anti-diabetic as well as the side effects (El-Mir et al., 2000; 


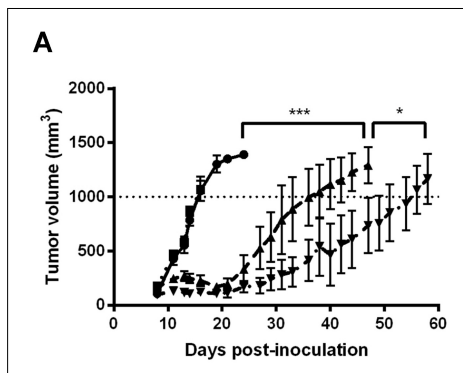

D

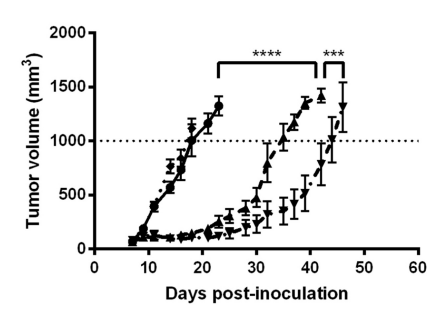

B

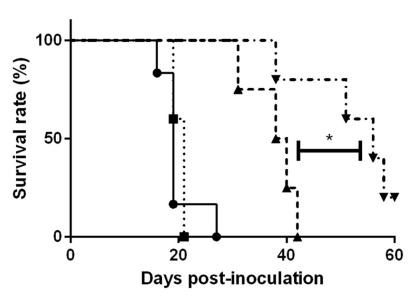

E

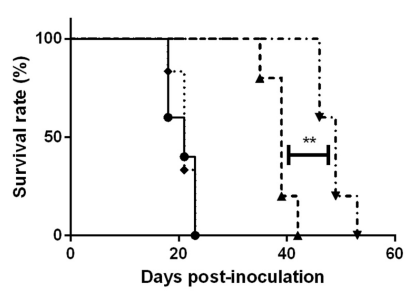

C

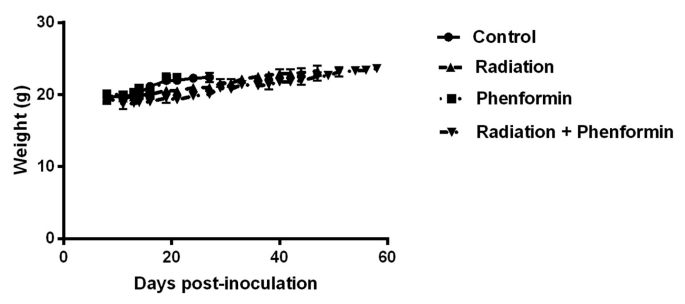

$\mathbf{F}$

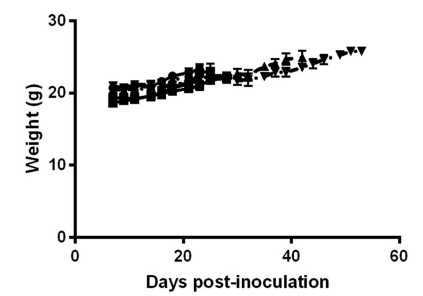

$\rightarrow$ Control

- Radiation

-. Metformin

$\rightarrow$ Radiation + Metformin

FIGURE 5 | Metformin and phenformin enhance radioresponse of CT26 tumor. Metformin (300 mg/kg) and phenformin (200 mg/kg) were administered through oral gavage for 10 times to CT26 tumor-bearing mice, and radiation (9Gy) was delivered on the first day of treatment. Tumor growth delay in mice treated with radiation, phenformin or Metformin, and the combination (A,D). Survival curves of mice euthanized when tumor diameter reached $15 \mathrm{~mm}$ (B,E). Assessment of toxicity by body weight loss (C,F). An ordinary one-way ANOVA with Tukey's multiple comparisons test and a survival analysis was used to calculate statistics: * $p<0.05$, ${ }^{* *} p<0.01,{ }^{* * *} p<0.001,{ }^{* * * *} p<0.0001$.

Owen et al., 2000). In our study, we found that at a $\mu \mathrm{M}$ level, phenformin profoundly inhibited complex I activity in CRC cells with about 100-fold more potency than metformin $(\mathrm{mM})$. This enormous difference between these two compounds is most likely due to the distinct physical properties. They share a similar chemical structure, however, phenformin is lipophilic and can readily permeate cells while metformin is hydrophilic, requiring an organic cation transporter (OCT) for cell entry. In normal conditions, OCTs are highly expressed in enterocytes in the intestine and hepatocytes in the liver, reflecting the pharmacokinetics of metformin that is absorbed in the intestine and then delivered to the liver to slow down glycogenesis (He and Wondisford, 2015). Besides normal tissue, OCTs are overexpressed in malignancies such as colorectal, prostate, and ovarian cancer (Segal et al., 2011; Tashiro et al., 2014; Obinata et al., 2016), and the downregulation of OCTs limits the antineoplastic effects of metformin (Segal et al., 2011). In our set up, the OCT expression although was not examined, it has been previously proved to be expressed in the applied cell lines (Kitada et al., 2008; Wheaton et al., 2014).

Clinically, the standard doses of metformin for diabetic patients are 1,500-2,500 $\mathrm{mg}$ per day with maximum plasma levels in the range of 10-25 $\mu \mathrm{M}$ (Graham et al., 2011). As aforementioned, metformin exerts its anti-cancer effects through multiple mechanisms, some of the actions are well attainable at $\mu \mathrm{M}$ levels, such as activation of AMPK via phosphorylation of Thr172 subunit (He and Wondisford, 2015). However, the concentrations required inhibiting complex I in our and other studies are much higher than the plasma levels (Wheaton et al., 2014), making people question the clinical relevance of these findings. Somehow, metformin at $\mathrm{mM}$ levels are likely achievable in clinic in CRC owing to the specific location and characteristics. Firstly, in intestine where CRC is located, at standard doses for diabetic patients, metformin could reach $\mathrm{mM}$ levels, which is most probably due to the high expression of OCTs in enterocytes (Bailey et al., 2008). Of note, efforts have been made to augment the activity of metformin; by attaching a lipophilic substituent, inhibitory effect of metformin toward complex I is potentiated about 1000-fold (Kalyanaraman et al., 2017). Secondly, it has been shown that cancer cells exhibiting higher mitochondrial respiratory activity are more sensitive to biguanide drugs (Hsu et al., 2015). In CRC, in contrast to the dogma that glycolysis is the predominant metabolism in cancer cells, the rate of oxygen consumption is higher in carcinomas as compared with normal adjacent tissues (Kaldma et al., 2014). This indicates that a reversed Warburg effect may exist in colorectal carcinomas, which may lead to a more predisposition of CRC cells to biguanides. Finally, OCTs and complex I have both reported to be higher expressed in the cancerous than the matched normal tissue in CRC (Yokoo et al., 2008; Wallace et al., 2016), suggesting a possibility of a selective cytotoxicity of metformin to CRC cells.

In agreement with the different extent of inhibitory activity toward complex I, phenformin displayed stronger cytotoxicity to CRC cells than metformin, in line with the observation from others in melanoma, breast, prostate, and lung cancer cells (Miskimins et al., 2014). However, at a concentration below $9 \mathrm{mM}$ and $100 \mu \mathrm{M}$, respectively, for metformin and phenformin, for which were applied further to explore the radiosensitization, no cytotoxicity was observed. Seemingly, at these concentrations, inhibition of complex I caused decrease of ATP and increase of 
ROS, which normally lead to cytotoxicity, are compensated by upregulated glycolysis. Indeed, glucose deprivation or glycolysis inhibitors synergize the cytotoxic effect of metformin and phenformin (Wheaton et al., 2014).

Hypoxia, as a result of an imbalance between oxygen supply and consumption, is a common feature of solid tumors and a well-defined risk factor for radiotherapy failure (Gray et al., 1953). To date, numerous tools have been explored and developed to overcome hypoxia-induced radioresistance (Overgaard, 2007). However, their clinical application is hampered due to the practical limitations and toxicity. As an alternative approach, inhibition of oxygen consumption is proposed and demonstrated to be more efficient at alleviating hypoxia than increasing oxygen delivery (Secomb et al., 1995). Several drugs that target different components of the mitochondrial electron transport chain including complex I, II, III, and IV, indeed substantially counteracted hypoxic radioresistance via increased tumor oxygenation (Jordan et al., 2002; Crokart et al., 2007; Diepart et al., 2012; Jiang et al., 2013; Ashton et al., 2016). Glucocorticoids as an inhibitor of cytochrome c oxidase (complex IV) significantly promoted tumor oxygenation and enhanced the tumor radiosensitivity although administration of glucocorticoids somewhat decreased perfusion (Crokart et al., 2007). Insulin, as an inducer of nitric oxide that inhibits complex II, increased both tumor oxygenation and radioresponse in a liver and fibrocarcoma mouse tumors, with more tumor growth delay than carbogen breathing (95\% $\mathrm{O}_{2}, 5 \% \mathrm{CO}_{2}$ ) (Jordan et al., 2002). Metformin is shown to reduce tumor hypoxia fraction and enhance radioresponse as well through inhibition of tumor cell respiration while little is known about phenformin (Zannella et al., 2013). In line, we found that metformin and phenformin as complex I inhibitors decreased oxygen consumption and enhanced hypoxic radiosensitivity of CRC cells by about 2 -fold at doses of $9 \mathrm{mM}$ and $100 \mu \mathrm{M}$, respectively. Recently, mitochondrial glycerophosphate dehydrogenase (MGPDH), a key enzyme connecting oxidative phosphorylation and glycolysis, is shown to be inhibited by metformin (Madiraju et al., 2014). Interestingly, this effect leads to reduction of mitochondrial respiration in thyroid cancer-derived cell lines (Thakur et al., 2018). As a next step, it could be important to explore whether MGPDH inhibition is attributable to the hypoxic radiosensitization of metformin and phenformin.

Next to hypoxic radiosenstization, both metformin and phenformin have been reported to radiosensitize aerobic tumor cells, including breast, lung, and pancreatic cancer cells (Storozhuk et al., 2013; Zhang et al., 2014; Wang et al., 2015a,b). The proposed mechanisms are inhibition of mTOR via activation of AMPK or upregulation of ROS production via disruption of antioxidant systems. mTOR inhibitors, such as rapamycin (Nagata et al., 2010), BEZ235 (Chang et al., 2014), and TAK228 (Miyahara et al., 2017), and pharmacological ROS insults, such as buthionine sulphoximine (Leung et al., 1993), auranofin (Wang et al., 2017), and piperlongumine (Matschke et al., 2016), overcame intrinsic radioresistance, leading to delayed tumor growth in different tumor models. Nevertheless, in the CRC cells used in our study, we found no evidence that metformin or phenformin affects intrinsic radiosensitivity. The intrinsic radiosensitivity of tumor cells is influenced by various factors, including DNA repair capacity, redox protein network, and expression patterns of oncogenes and tumor suppresses genes. The active extent of these factors differs in varying types of cancer, which might interpret the distinct response.

In concordance with the in vitro hypoxic radiosensitization, in syngeneic tumors, we found that metformin enhanced radioresponse and significantly increased the medium survival rate of tumor-bearing mice, which is in line with the findings in a CRC xenograft model that metformin was administered $30 \mathrm{~min}$ prior to radiotherapy (Zannella et al., 2013). In our study, the radiosensitizing effect was observed at a dose that is close to the maximal recommended safe dose via oral gavage (Song et al., 2012); in the other study, although it was $25 \%$ of the daily dose of patients, metformin was injected intravenously that could markedly boost plasma levels (Zannella et al., 2013). It is worth mentioning that different tumor models possess diverse characteristics, thus their susceptibility toward metformin and phenformin may differ. In spheroids of hypopharyngeal carcinoma cells and CRC cells, metformin and phenformin inhibited cellular respiration and reduced hypoxic fractions, but were ineffective in spheroids of lung cancer cells (Ashton et al., 2016). In addition, in a lung cancer xenograft model, although intraperitoneal injection of metformin and phenformin resulted in higher plasma concentrations than oral administration, the hypoxic fraction of the tumor was not affected by the treatments (Iversen et al., 2017). Whereas using the same lung cancer xenograft, phenformin improved radioresponse with a mechanism ascribed to activation of the AMPK pathway (Wang et al., 2015a). Hereby, our study is the first, to the best of our knowledge, to provide evidence that phenformin could radiosensitize colorectal tumor with a mechanism linked to a decrease of OCR via inhibition of complex I. We cannot exclude that other mechanisms might implicate in, such as boosting tumor immune surveillance. Metformin and phenformin are indeed known to inhibit the activity of pro-tumor MDSC and macrophages, and to enhance the generation of anti-tumor memory CD8+T-cell, consequently improving the efficacy of anti-cancer vaccine and PD-1 blockade (Eikawa et al., 2015; Kim et al., 2017; Scharping et al., 2017). Our preliminary data suggest that phenformin could deplete MDSC, while the effect of metformin and phenformin on memory CD8+T-cell is still under investigation.

Currently, dozens of clinical trials with metformin are initiated focusing mainly on breast and prostate cancer. About 10 on-going prospective phase II clinical trials are launched to explore whether metformin may improve therapy outcomes or lower CRC incidence in patients without diabetes. Among them, three address neoadjuvant metformin incorporation of radiotherapy in locally advanced rectal cancer (NCT02437656, NCT03053544). One is initiated in our institution, where metformin is combined with neoadjuvant chemoradiation to improve tumor radioresponse and patient outcome for locally advanced cT3-4 rectal cancer (EudraCT number: 2017-00081450). Unlike metformin, the research wave of phenformin is just beginning with only one clinical trial initiated in 
which phenformin is combined with chemotherapeutic agents to treat metastatic melanoma (NCT03026517). Intriguingly, phenformin combined with non-pharmacological measures (rational nutrition, moderate physical activity and so forth) has been prescribed to breast and gastrointestinal cancer patients, aiming to rehabilitate the metabolism in 1970-1990 (Dilman et al., 1988; Berstein, 2010). After 10 years follow-up, the studies unveiled that this regimen is associated with improved relapse-free and total survival. The downside of these studies was the absence of proper randomization; nevertheless, they provide the first clinical evidence that phenformin may improve the outcome of cancer patients. Admittedly, phenformin has a predisposition to cause lactic acidosis in diabetic patients with renal failure, however, this side effect may be manageable by prescreening kidney function. Taken into account that the duration of phenformin administration in cancer patients would be shorter from its prior clinical use for diabetes, thus there is a possibility of a decreased incidence of severe side effects. In addition, it was reported that supplementation of glycolysis inhibitor with phenformin might avoid the risk of lactic acidosis and in the same time potentiate the antitumor effect (Lea et al., 2011; Gravel et al., 2014; Miskimins et al., 2014).

Taken together, in this study, we demonstrate that metformin and phenformin inhibit mitochondrial complex I and thereby block cellular respiration of CRC cells, leading to enhanced radioresponse. The radiosensitizing effect of metformin has been acknowledged with clinical trials initiated in combination with radiotherapy, while for phenformin it is still a completely blank page in clinical settings. Based on our and other's findings, phenformin next to metformin merits clinical investigation as well and may represent an effective and inexpensive means to improve radiotherapy outcome.

\section{REFERENCES}

Andrzejewski, S., Gravel, S. P., Pollak, M., and St-Pierre, J. (2014). Metformin directly acts on mitochondria to alter cellular bioenergetics. Cancer Metab. 2:12. doi: 10.1186/2049-3002-2-12

Ashton, T. M., Fokas, E., Kunz-Schughart, L. A., Folkes, L. K., Anbalagan, S., Huether, M., et al. (2016). The anti-malarial atovaquone increases radiosensitivity by alleviating tumour hypoxia. Nat. Commun. 7:12308 doi: $10.1038 /$ ncomms 12308

Bailey, C. J., Wilcock, C., and Scarpello, J. H. (2008). Metformin and the intestine. Diabetologia 51, 1552-1553. doi: 10.1007/s00125-008-1053-5

Berstein, L. M. (2010). Modern approach to metabolic rehabilitation of cancer patients: biguanides (phenformin and metformin) and beyond. Future Oncol. 6, 1313-1323. doi: 10.2217/fon.10.87

Boukalova, S., Stursa, J., Werner, L., Ezrova, Z., Cerny, J., Bezawork-Geleta, A., et al. (2016). Mitochondrial targeting of metformin enhances its activity against pancreatic cancer. Mol. Cancer Ther. 15, 2875-2886. doi: 10.1158/1535-7163. mct-15-1021

Chae, Y. K., Arya, A., Malecek, M. K., Shin, D. S., Carneiro, B., Chandra, S., et al. (2016). Repurposing metformin for cancer treatment: current clinical studies. Oncotarget 7, 40767-40780. doi: 10.18632/oncotarget. 8194

Chang, L., Graham, P. H., Hao, J., Ni, J., Bucci, J., Cozzi, P. J., et al. (2014). $\mathrm{PI} 3 \mathrm{~K} / \mathrm{Akt} / \mathrm{mTOR}$ pathway inhibitors enhance radiosensitivity in radioresistant prostate cancer cells through inducing apoptosis, reducing autophagy, suppressing NHEJ and HR repair pathways. Cell Death Dis. 5:e1437. doi: 10. 1038/cddis.2014.415

\section{ETHICS STATEMENT}

This study was carried out in accordance with the recommendations of the Ethical Committee for use of laboratory animals of the Vrije Universiteit Brussel. The protocol was approved by the Ethical Committee for use of laboratory animals of the Vrije Universiteit Brussel.

\section{AUTHOR CONTRIBUTIONS}

SdM and HJ conceived and designed the experiments, and wrote the manuscript. SdM and KL performed the experiments. CC and EB designed and helped performing the Seahorse experiments. TG helped with the radiation of the cells and mice. HW, ID, and $\mathrm{VV}$ revised the manuscript critically for important intellectual content. MDR and OF supervised the study and helped writing the manuscript. All authors discussed the results and contributed to the final manuscript.

\section{FUNDING}

This work was supported by grant from the Kom Op Tegen Kanker.

\section{SUPPLEMENTARY MATERIAL}

The Supplementary Material for this article can be found online at: https://www.frontiersin.org/articles/10.3389/fphar. 2018.01073/full\#supplementary-material

Corbet, C., Pinto, A., Martherus, R., Santiago de Jesus, J. P., Polet, F., and Feron, O. (2016). Acidosis drives the reprogramming of fatty acid metabolism in cancer cells through changes in mitochondrial and histone acetylation. Cell Metab. 24, 311-323. doi: 10.1016/j.cmet.2016.07.003

Crokart, N., Jordan, B. F., Baudelet, C., Cron, G. O., Hotton, J., Radermacher, K., et al. (2007). Glucocorticoids modulate tumor radiation response through a decrease in tumor oxygen consumption. Clin. Cancer Res. 13(2 Pt 1), 630-635. doi: 10.1158/1078-0432.ccr-06-0802

Decensi, A., Puntoni, M., Goodwin, P., Cazzaniga, M., Gennari, A., Bonanni, B., et al. (2010). Metformin and cancer risk in diabetic patients: a systematic review and meta-analysis. Cancer Prev. Res. 3, 1451-1461. doi: 10.1158/1940-6207. capr-10-0157

Diepart, C., Karroum, O., Magat, J., Feron, O., Verrax, J., Calderon, P. B., et al. (2012). Arsenic trioxide treatment decreases the oxygen consumption rate of tumor cells and radiosensitizes solid tumors. Cancer Res. 72, 482-490. doi: 10.1158/0008-5472.can-11-1755

Dilman, V. M., Berstein, L. M., Yevtushenko, T. P., Tsyrlina, Y. V., Ostroumova, M. N., Bobrov, Y. F., et al. (1988). Preliminary evidence on metabolic rehabilitation of cancer patients. Arch. Geschwulstforsch. 58, 175-183.

Eikawa, S., Nishida, M., Mizukami, S., Yamazaki, C., Nakayama, E., and Udono, H. (2015). Immune-mediated antitumor effect by type 2 diabetes drug, metformin. Proc. Natl. Acad. Sci. U.S.A. 112, 1809-1814. doi: 10.1073/pnas.1417636112

El-Mir, M. Y., Nogueira, V., Fontaine, E., Averet, N., Rigoulet, M., and Leverve, X. (2000). Dimethylbiguanide inhibits cell respiration via an indirect effect targeted on the respiratory chain complex I. J. Biol. Chem. 275, 223-228.

Goodwin, P. J., Thompson, A. M., and Stambolic, V. (2012). Diabetes, metformin, and breast cancer: lilac time? J. Clin. Oncol. 30, 2812-2814. 
Graham, G. G., Punt, J., Arora, M., Day, R. O., Doogue, M. P., Duong, J. K., et al. (2011). Clinical pharmacokinetics of metformin. Clin. Pharmacokinet. 50, 81-98. doi: 10.2165/11534750-000000000-00000

Gravel, S. P., Hulea, L., Toban, N., Birman, E., Blouin, M. J., Zakikhani, M., et al. (2014). Serine deprivation enhances antineoplastic activity of biguanides. Cancer Res. 74, 7521-7533. doi: 10.1158/0008-5472.can-14-2643-t

Gray, L. H., Conger, A. D., Ebert, M., Hornsey, S., and Scott, O. C. (1953). The concentration of oxygen dissolved in tissues at the time of irradiation as a factor in radiotherapy. Br. J. Radiol. 26, 638-648. doi: 10.1259/0007-1285-26-31 $2-638$

Haugrud, A. B., Zhuang, Y., Coppock, J. D., and Miskimins, W. K. (2014). Dichloroacetate enhances apoptotic cell death via oxidative damage and attenuates lactate production in metformin-treated breast cancer cells. Breast Cancer Res. Treat. 147, 539-550. doi: 10.1007/s10549-0143128-y

He, L., and Wondisford, F. E. (2015). Metformin action: concentrations matter. Cell Metab. 21, 159-162. doi: 10.1016/j.cmet.2015.01.003

Hsu, C. C., Wu, L. C., Hsia, C. Y., Yin, P. H., Chi, C. W., Yeh, T. S., et al. (2015). Energy metabolism determines the sensitivity of human hepatocellular carcinoma cells to mitochondrial inhibitors and biguanide drugs. Oncol. Rep. 34, 1620-1628. doi: 10.3892/or.2015.4092

Iversen, A. B., Horsman, M. R., Jakobsen, S., Jensen, J. B., Garm, C., Jessen, N., et al. (2017). Results from (11)C-metformin-PET scans, tissue analysis and cellular drug-sensitivity assays questions the view that biguanides affects tumor respiration directly. Sci. Rep. 7:9436. doi: 10.1038/s41598-017-10010-z

Janssens, M. Y., Van den Berge, D. L., Verovski, V. N., Monsaert, C., and Storme, G. A. (1998). Activation of inducible nitric oxide synthase results in nitric oxide-mediated radiosensitization of hypoxic EMT-6 tumor cells. Cancer Res. 58, 5646-5648.

Janssens, M. Y., Verovski, V. N., Van den Berge, D. L., Monsaert, C., and Storme, G. A. (1999). Radiosensitization of hypoxic tumour cells by S-nitroso$\mathrm{N}$-acetylpenicillamine implicates a bioreductive mechanism of nitric oxide generation. Br. J. Cancer 79, 1085-1089. doi: 10.1038/sj.bjc.6690173

Jiang, H., De Ridder, M., Verovski, V. N., Sonveaux, P., Jordan, B. F., Law, K., et al. (2010). Activated macrophages as a novel determinant of tumor cell radioresponse: the role of nitric oxide-mediated inhibition of cellular respiration and oxygen sparing. Int. J. Radiat. Oncol. Biol. Phys. 76, 1520-1527. doi: 10.1016/j.ijrobp.2009.10.047

Jiang, H., Verovski, V. N., Leonard, W., Law, K. L., Vermeersch, M., Storme, G., et al. (2013). Hepatocytes determine the hypoxic microenvironment and radiosensitivity of colorectal cancer cells through production of nitric oxide that targets mitochondrial respiration. Int. J. Radiat. Oncol. Biol. Phys. 85, 820-827. doi: 10.1016/j.ijrobp.2012.07.2359

Jordan, B. F., Gregoire, V., Demeure, R. J., Sonveaux, P., Feron, O., O’Hara, J., et al. (2002). Insulin increases the sensitivity of tumors to irradiation: involvement of an increase in tumor oxygenation mediated by a nitric oxide-dependent decrease of the tumor cells oxygen consumption. Cancer Res. 62, 3555-3561.

Kaldma, A., Klepinin, A., Chekulayev, V., Mado, K., Shevchuk, I., Timohhina, N., et al. (2014). An in situ study of bioenergetic properties of human colorectal cancer: the regulation of mitochondrial respiration and distribution of flux control among the components of ATP synthasome. Int. J. Biochem. Cell Biol. 55, 171-186. doi: 10.1016/j.biocel.2014.09.004

Kalyanaraman, B., Cheng, G., Hardy, M., Ouari, O., Sikora, A., Zielonka, J., et al. (2017). Modified metformin as a more potent anticancer drug: mitochondrial inhibition, redox signaling, antiproliferative effects and future EPR studies. Cell Biochem. Biophys. 75, 311-317. doi: 10.1007/s12013-017-0796-3

Kim, S. H., Li, M., Trousil, S., Zhang, Y., Pasca di Magliano, M., Swanson, K. D., et al. (2017). Phenformin inhibits myeloid-derived suppressor cells and enhances the anti-tumor activity of PD-1 blockade in melanoma. J. Invest. Dermatol. 137, 1740-1748. doi: 10.1016/j.jid.2017.03.033

Kitada, N., Takara, K., Minegaki, T., Itoh, C., Tsujimoto, M., Sakaeda, T., et al. (2008). Factors affecting sensitivity to antitumor platinum derivatives of human colorectal tumor cell lines. Cancer Chemother. Pharmacol. 62, 577-584. doi: 10.1007/s00280-007-0640-3

Lea, M. A., Chacko, J., Bolikal, S., Hong, J. Y., Chung, R., Ortega, A., et al. (2011). Addition of 2-deoxyglucose enhances growth inhibition but reverses acidification in colon cancer cells treated with phenformin. Anticancer. Res. 31, $421-426$.
Leung, S. W., Mitchell, J. B., al-Nabulsi, I., Friedman, N., Newsome, J., Belldegrun, A., et al. (1993). Effect of L-buthionine sulfoximine on the radiation response of human renal carcinoma cell lines. Cancer 71, 2276-2285.

Lin, A., and Maity, A. (2015). Molecular pathways: a novel approach to targeting hypoxia and improving radiotherapy efficacy via reduction in oxygen demand. Clin. Cancer Res. 21, 1995-2000. doi: 10.1158/1078-0432.ccr-14-0858

Lin, H., Li, N., He, H., Ying, Y., Sunkara, S., Luo, L., et al. (2015). AMPK inhibits the stimulatory effects of TGF-beta on Smad2/3 activity, cell migration, and epithelial-to-mesenchymal transition. Mol. Pharmacol. 88, 1062-1071. doi: 10. 1124/mol.115.099549

Liu, Z., Ren, L., Liu, C., Xia, T., Zha, X., and Wang, S. (2015). Phenformin induces cell cycle change, apoptosis, and mesenchymal-epithelial transition and regulates the AMPK/mTOR/p70s6k and MAPK/ERK pathways in breast cancer cells. PLoS One 10:e0131207. doi: 10.1371/journal.pone.0131207

Madiraju, A. K., Erion, D. M., Rahimi, Y., Zhang, X. M., Braddock, D. T., Albright, R. A., et al. (2014). Diabetes, metformin, and breast cancer: lilac time Metformin suppresses gluconeogenesis by inhibiting mitochondrial glycerophosphate dehydrogenase. Nature 510, 542-546. doi: 10.1038/nature13270

Mallik, R., and Chowdhury, T. A. (2018). Metformin in cancer. Diabetes Res. Clin. Pract. doi: 10.1016/j.diabres.2018.05.023 [Epub ahead of print].

Matschke, J., Riffkin, H., Klein, D., Handrick, R., Ludemann, L., Metzen, E., et al. (2016). Targeted inhibition of glutamine-dependent glutathione metabolism overcomes death resistance induced by chronic cycling hypoxia. Antioxid. Redox. Signal. 25, 89-107. doi: 10.1089/ars.2015.6589

Matsuzaki, S., and Humphries, K. M. (2015). Selective inhibition of deactivated mitochondrial complex I by biguanides. Biochemistry 54, 2011-2021. doi: 10 . 1021/bi501473h

Miskimins, W. K., Ahn, H. J., Kim, J. Y., Ryu, S., Jung, Y. S., and Choi, J. Y. (2014). Synergistic anti-cancer effect of phenformin and oxamate. PLoS One 9:e85576. doi: 10.1371/journal.pone.0085576

Miyahara, H., Yadavilli, S., Natsumeda, M., Rubens, J. A., Rodgers, L., Kambhampati, M., et al. (2017). The dual mTOR kinase inhibitor TAK228 inhibits tumorigenicity and enhances radiosensitization in diffuse intrinsic pontine glioma. Cancer Lett. 400, 110-116. doi: 10.1016/j.canlet.2017.04.019

Momcilovic, M., McMickle, R., Abt, E., Seki, A., Simko, S. A., Magyar, C., et al. (2015). Heightening energetic stress selectively targets LKB1-deficient nonsmall cell lung cancers. Cancer Res. 75, 4910-4922. doi: 10.1158/0008-5472.can15-0797

Nagata, Y., Takahashi, A., Ohnishi, K., Ota, I., Ohnishi, T., Tojo, T., et al. (2010). Effect of rapamycin, an mTOR inhibitor, on radiation sensitivity of lung cancer cells having different p53 gene status. Int. J. Oncol. 37, 1001-1010.

Navarro, P., Bueno, M. J., Zagorac, I., Mondejar, T., Sanchez, J., Mouron, S., et al. (2016). Targeting tumor mitochondrial metabolism overcomes resistance to antiangiogenics. Cell Rep. 15, 2705-2718. doi: 10.1016/j.celrep.2016.05.052

Obinata, D., Takayama, K., Fujiwara, K., Suzuki, T., Tsutsumi, S., Fukuda, N., et al. (2016). Targeting Octl genomic function inhibits androgen receptor signaling and castration-resistant prostate cancer growth. Oncogene 35, 6350-6358. doi: 10.1038/onc.2016.171

Orecchioni, S., Reggiani, F., Talarico, G., Mancuso, P., Calleri, A., Gregato, G., et al. (2015). The biguanides metformin and phenformin inhibit angiogenesis, local and metastatic growth of breast cancer by targeting both neoplastic and microenvironment cells. Int. J. Cancer 136, E534-E544. doi: 10.1002/ijc.29193

Overgaard, J. (2007). Hypoxic radiosensitization: adored and ignored. J. Clin. Oncol. 25, 4066-4074. doi: 10.1200/jco.2007.12.7878

Owen, M. R., Doran, E., and Halestrap, A. P. (2000). Evidence that metformin exerts its anti-diabetic effects through inhibition of complex 1 of the mitochondrial respiratory chain. Biochem. J. 348(Pt 3), 607-614.

Paul, M. K., Kumar, R., and Mukhopadhyay, A. K. (2008). Dithiothreitol abrogates the effect of arsenic trioxide on normal rat liver mitochondria and human hepatocellular carcinoma cells. Toxicol. Appl. Pharmacol. 226, 140-152. doi: 10.1016/j.taap.2007.09.020

Pernicova, I., and Korbonits, M. (2014). Metformin-mode of action and clinical implications for diabetes and cancer. Nat. Rev. Endocrinol. 10, 143-156. doi: 10.1038/nrendo.2013.256

Petrachi, T., Romagnani, A., Albini, A., Longo, C., Argenziano, G., Grisendi, G., et al. (2017). Therapeutic potential of the metabolic modulator phenformin in targeting the stem cell compartment in melanoma. Oncotarget 8, 6914-6928. doi: 10.18632/oncotarget.14321 
Quinn, B. J., Kitagawa, H., Memmott, R. M., Gills, J. J., and Dennis, P. A. (2013). Repositioning metformin for cancer prevention and treatment. Trends Endocrinol. Metab. 24, 469-480. doi: 10.1016/j.tem.2013.05.004

Scharping, N. E., Menk, A. V., Whetstone, R. D., Zeng, X., and Delgoffe, G. M. (2017). Efficacy of PD-1 blockade is potentiated by metformin-induced reduction of tumor hypoxia. Cancer Immunol. Res. 5, 9-16. doi: 10.1158/23266066.cir-16-0103

Secomb, T. W., Hsu, R., Ong, E. T., Gross, J. F., and Dewhirst, M. W. (1995). Analysis of the effects of oxygen supply and demand on hypoxic fraction in tumors. Acta Oncol. 34, 313-316.

Segal, E. D., Yasmeen, A., Beauchamp, M. C., Rosenblatt, J., Pollak, M., and Gotlieb, W. H. (2011). Relevance of the OCT1 transporter to the antineoplastic effect of biguanides. Biochem. Biophys. Res. Commun. 414, 694-699. doi: 10.1016/j.bbrc. 2011.09.134

Shackelford, D. B., Abt, E., Gerken, L., Vasquez, D. S., Seki, A., Leblanc, M., et al. (2013). LKB1 inactivation dictates therapeutic response of non-small cell lung cancer to the metabolism drug phenformin. Cancer Cell 23, 143-158. doi: $10.1016 /$ j.ccr.2012.12.008

Song, C. W., Lee, H., Dings, R. P., Williams, B., Powers, J., Santos, T. D., et al. (2012). Metformin kills and radiosensitizes cancer cells and preferentially kills cancer stem cells. Sci. Rep. 2:362. doi: 10.1038/srep00362

Storozhuk, Y., Hopmans, S. N., Sanli, T., Barron, C., Tsiani, E., Cutz, J. C., et al. (2013). Metformin inhibits growth and enhances radiation response of nonsmall cell lung cancer (NSCLC) through ATM and AMPK. Br. J. Cancer 108, 2021-2032. doi: 10.1038/bjc.2013.187

Tashiro, A., Tatsumi, S., Takeda, R., Naka, A., Matsuoka, H., Hashimoto, Y., et al. (2014). High expression of organic anion transporter 2 and organic cation transporter 2 is an independent predictor of good outcomes in patients with metastatic colorectal cancer treated with FOLFOX-based chemotherapy. Am. J. Cancer Res. 4, 528-536.

Thakur, S., Daley, B., Gaskins, K., Vasko, V. V., Boufraqech, M., Patel, D., et al. (2018). Metformin targets mitochondrial glycerophosphate dehydrogenase to control rate of oxidative phosphorylation and growth of thyroid cancer in vitro and in vivo. Clin. Cancer Res. 24, 4030-4043. doi: 10.1158/1078-0432.ccr-173167

Velez, J., Pan, R., Lee, J. T., Enciso, L., Suarez, M., Duque, J. E., et al. (2016). Biguanides sensitize leukemia cells to ABT-737-induced apoptosis by inhibiting mitochondrial electron transport. Oncotarget 7, 51435-51449. doi: 10.18632/ oncotarget.9843

Wallace, L., Mehrabi, S., Bacanamwo, M., Yao, X., and Aikhionbare, F. O. (2016). Expression of mitochondrial genes MT-ND1, MT-ND6, MT-CYB, MT-COI, MT-ATP6, and 12S/MT-RNR1 in colorectal adenopolyps. Tumour Biol. 37, 12465-12475. doi: 10.1007/s13277-016-5101-3
Wang, H., Bouzakoura, S., de Mey, S., Jiang, H., Law, K., Dufait, I., et al. (2017). Auranofin radiosensitizes tumor cells through targeting thioredoxin reductase and resulting overproduction of reactive oxygen species. Oncotarget 8, 3572835742. doi: 10.18632/oncotarget.16113

Wang, J., Xia, S., and Zhu, Z. (2015a). Synergistic effect of phenformin in non-small cell lung cancer (NSCLC) ionizing radiation treatment. Cell Biochem. Biophys. 71, 513-518. doi: 10.1007/s12013-014-0283-Z

Wang, Z., Lai, S. T., Ma, N. Y., Deng, Y., Liu, Y., Wei, D. P., et al. (2015b). Radiosensitization of metformin in pancreatic cancer cells via abrogating the G2 checkpoint and inhibiting DNA damage repair. Cancer Lett. 369, 192-201. doi: 10.1016/j.canlet.2015.08.015

Wheaton, W. W., Weinberg, S. E., Hamanaka, R. B., Soberanes, S., Sullivan, L. B., Anso, E., et al. (2014). Metformin inhibits mitochondrial complex I of cancer cells to reduce tumorigenesis. eLife 3:e02242. doi: 10.7554/eLife. 02242

Yokoo, S., Masuda, S., Yonezawa, A., Terada, T., Katsura, T., and Inui, K. (2008). Significance of organic cation transporter 3 (SLC22A3) expression for the cytotoxic effect of oxaliplatin in colorectal cancer. Drug Metab. Dispos. 36, 2299-2306. doi: 10.1124/dmd.108.023168

Zannella, V. E., Dal Pra, A., Muaddi, H., McKee, T. D., Stapleton, S., Sykes, J., et al. (2013). Reprogramming metabolism with metformin improves tumor oxygenation and radiotherapy response. Clin. Cancer Res. 19, 6741-6750. doi: 10.1158/1078-0432.ccr-13-1787

Zhang, Y., Storr, S. J., Johnson, K., Green, A. R., Rakha, E. A., Ellis, I. O., et al. (2014). Involvement of metformin and AMPK in the radioresponse and prognosis of luminal versus basal-like breast cancer treated with radiotherapy. Oncotarget 5, 12936-12949. doi: 10.18632/oncotarget.2683

Zhou, X., Chen, J., Yi, G., Deng, M., Liu, H., Liang, M., et al. (2016). Metformin suppresses hypoxia-induced stabilization of HIF-1alpha through reprogramming of oxygen metabolism in hepatocellular carcinoma. Oncotarget 7, 873-884. doi: 10.18632/oncotarget.6418

Conflict of Interest Statement: The authors declare that the research was conducted in the absence of any commercial or financial relationships that could be construed as a potential conflict of interest.

Copyright (c) 2018 de Mey, Jiang, Corbet, Wang, Dufait, Law, Bastien, Verovski, Gevaert, Feron and De Ridder. This is an open-access article distributed under the terms of the Creative Commons Attribution License (CC BY). The use, distribution or reproduction in other forums is permitted, provided the original author(s) and the copyright owner(s) are credited and that the original publication in this journal is cited, in accordance with accepted academic practice. No use, distribution or reproduction is permitted which does not comply with these terms. 